\title{
Genome-wide analysis suggests the importance of vascular processes and neuroinflammation in late- life antidepressant response
}

Victoria S. Marshe $\mathbb{1}^{1,2}$, Malgorzata Maciukiewicz ${ }^{3}$, Anne-Christin Hauschild ${ }^{4}$, Farhana Islam²,5, Li Qin², Arun K. Tiwari², Etienne Sibille $\mathbb{1}^{2,5,6}$, Daniel M. Blumberger ${ }^{1,2,6}$, Jordan F. Karp ${ }^{7}$, Alastair J. Flint ${ }^{1,6,8}$, Gustavo Turecki @9

Raymond W. Lam (10 ${ }^{10}$, Roumen V. Milev ${ }^{11}$, Benicio N. Frey (10 2,13, Susan Rotzinger ${ }^{8,14}$, Jane A. Foster ${ }^{13,14}$,

Sidney H. Kennedy $\mathbb{1 0}^{6,8,14,15}$, James L. Kennedy $\mathbb{1 0}^{1,2,6}$, Benoit H. Mulsant ${ }^{1,2,6}$, Charles F. Reynolds III7 , Eric J. Lenze ${ }^{16}$ and

Daniel J. Müller $\mathbb{D}^{1,2,5,6}$

\begin{abstract}
Antidepressant outcomes in older adults with depression is poor, possibly because of comorbidities such as cerebrovascular disease. Therefore, we leveraged multiple genome-wide approaches to understand the genetic architecture of antidepressant response. Our sample included 307 older adults ( $\geq 60$ years) with current major depression, treated with venlafaxine extended-release for 12 weeks. A standard genome-wide association study (GWAS) was conducted for post-treatment remission status, followed by in silico biological characterization of associated genes, as well as polygenic risk scoring for depression, neurodegenerative and cerebrovascular disease. The top-associated variants for remission status and percentage symptom improvement were PIEZO1 rs12597726 (OR= $\left.0.33[0.21,0.51], p=1.42 \times 10^{-6}\right)$ and intergenic rs6916777 (Beta $=14.03[8.47,19.59], p=1.25 \times 10^{-6}$ ), respectively. Pathway analysis revealed significant contributions from genes involved in the ubiquitin-proteasome system, which regulates intracellular protein degradation with has implications for inflammation, as well as atherosclerotic cardiovascular disease ( $n=25$ of 190 genes, $p=8.03 \times 10^{-6}$, FDR-corrected $p=0.01$ ). Given the polygenicity of complex outcomes such as antidepressant response, we also explored 11 polygenic risk scores associated with risk for Alzheimer's disease and stroke. Of the 11 scores, risk for cardioembolic stroke was the second-best predictor of nonremission, after being male (Accuracy $=0.70[0.59,0.79]$, Sensitivity $=0.72$, Specificity $=0.67 ; p=2.45 \times 10^{-4}$ ).

Although our findings did not reach genome-wide significance, they point to previously-implicated mechanisms and provide support for the roles of vascular and inflammatory pathways in LLD. Overall, significant enrichment of genes involved in protein degradation pathways that may be impaired, as well as the predictive capacity of risk for cardioembolic stroke, support a link between late-life depression remission and risk for vascular dysfunction.
\end{abstract}

\section{Introduction}

Major Depressive Disorder (MDD) occurring in adults $\geq 60$ years is frequently referred to as Late-Life Depression

\footnotetext{
Correspondence: Daniel J. Müller (daniel.mueller@camh.ca)

${ }^{1}$ Institute of Medical Science, University of Toronto, Toronto, ON, Canada

${ }^{2}$ Campbell Family Mental Health Research Institute, Centre for Addiction and Mental Health, Toronto, ON, Canada

Full list of author information is available at the end of the article
}

(LDD) and has an annual prevalence estimate of $4 \%$ in community-dwelling older adults in the United States ${ }^{1}$. The multidimensional interaction between ageing and depression presents a challenge in the treatment of LLD, with a significant proportion $(>50 \%)$ of patients failing to achieve remission with antidepressant pharmacotherapy ${ }^{2}$. In particular, older adults who do not achieve LLD remission are at an increased risk of cognitive decline and

\section{(c) The Author(s) 2021}

(c) (i) Open Access This article is licensed under a Creative Commons Attribution 4.0 International License, which permits use, sharing, adaptation, distribution and reproduction c. in any medium or format, as long as you give appropriate credit to the original author(s) and the source, provide a link to the Creative Commons license, and indicate if changes were made. The images or other third party material in this article are included in the article's Creative Commons license, unless indicated otherwise in a credit line to the material. If material is not included in the article's Creative Commons license and your intended use is not permitted by statutory regulation or exceeds the permitted use, you will need to obtain permission directly from the copyright holder. To view a copy of this license, visit http://creativecommons.org/licenses/by/4.0/. 
dementia, possibly due to cerebrovascular disease cooccurring with depression ${ }^{3}$. Therefore, finding genetic markers to predict clinical outcomes may help identify novel drug targets and develop combinatorial pharmacogenomic treatment approaches and have been shown to improve depression outcomes in older adults ${ }^{4}$.

A growing body of evidence suggests that individual genetics contribute to antidepressant treatment outcomes and adverse drug events ${ }^{5}$. Genetic variability in cytochrome P450 (CYP) enzymes, which mediate the phase I oxidation of various antidepressants, has been associated with inter-individual differences in drug response and tolerability. In particular, CYP2D6, which metabolizes $50-60 \%$ of all antidepressants, appears to have the most actionable pharmacogenetic effect in older adults ${ }^{6}$. Although there are no specific CYP2D6 guidelines for antidepressant dosing in older adults, those with reduced enzyme function require lower doses, similarly to middleaged adults ${ }^{6}$. Furthermore, age-related changes to neurotransmitter systems, such as the serotonergic system, have also been noted ${ }^{7}$. Although there are no actionable guidelines for the serotonin transporter gene (SLC6A4), a modest level of evidence suggests that older adults carrying the 5-HTTLPR low function variant (S) respond worse to antidepressant pharmacotherapy than those with high functioning variants $(\mathrm{L} / \mathrm{L})^{6}$. Although these findings are inconsistent, the observed effects of 5-HTTLPR may be similar to those in middle-aged adults, particularly of European-ancestry ${ }^{8}$.

Given the biological overlap between LLD, neurodegenerative disease (e.g. Alzheimer's disease), and cerebrovascular disease, there may be common risk pathways also contributing to antidepressant non-response ${ }^{9-11}$. A common process overlapping these diseases is neuroinflammation. Notably, antidepressants also decrease inflammation, and conversely, anti-inflammatory treatments may alleviate depressive symptoms ${ }^{12,13}$. The inflammation hypothesis of depression postulates that excessive inflammatory cascades result in neurotoxicity and neuronal death in key brain regions, such as the hippocampus, contributing to depressive symptoms ${ }^{14}$. These effects coincide with Alzheimer's disease neuropathology, whereby the excessive microglial response to the presence of amyloid plaques results in hippocampal atrophy ${ }^{15}$. Similarly, the neuroinflammatory and neurotoxic processes associated with cerebrovascular disease are observed in depressed older adults. For example, an increased burden of ischemic brain lesions (i.e. white matter hyperintensities) has been observed in neuroimaging studies of $L L D^{16}$. Furthermore, there is an increased frequency of depression in individuals with cerebrovascular disease ${ }^{11}$.

This co-prevalence of cerebrovascular disease and depression supports the vascular depression hypothesis, which posits that ischemic lesions in frontostriatal regions contribute to cognitive dysfunction, depressed mood and treatment resistance ${ }^{11,16}$. Evidence from structural magnetic resonance imaging has shown that ischemic lesions, known as white matter hyperintensities (WHMs), are associated with and predict the onset of depression ${ }^{17}$. Supporting molecular evidence suggests that vulnerabilities in multiple pathways contribute to the etiology of vascular pathology, including dysregulation of the hypothalamic-pituitary-adrenal axis, endothelial function, atherosclerosis and microglial activation ${ }^{11}$. As such, this bidirectional relationship between vascular and neurodegeneration processes underscores the importance of the risk pathways both for LLD risk and antidepressant nonresponse. As such, we are interested in identifying genetic variants across enriched in vulnerable inflammatory ${ }^{18}$, neurodegenerative and vascular pathways, which may inform molecular targets or pathways involved in the treatment response of LLD. This project has two discovery aims. First, to describe the first genome-wide study of antidepressant response in depressed older adults. Our second aim was to present comprehensive post-GWAS analyses investigating both inflammatory and vascular pathways involved in antidepressant response and the predictive potential of polygenic risk scores.

\section{Methods \\ Discovery cohort-IRL-GREY}

Our sample consisted of adults $\geq 60$ years from the NIHfunded clinical trial IRL-GREY (Incomplete Response in Late-Life Depression: Getting to Remission; NCT00892047). Participants received open-label venlafaxine $\left(37.5 \mathrm{mg} /\right.$ day, up to $300 \mathrm{mg} /$ day) for 12 weeks ${ }^{19}$. Inclusion criteria included a DSM-IV diagnosis of MDD with at least moderately severe symptoms as defined by a Montgomery-Åsberg Depression Rating Scale ${ }^{20}$ (MADRS) score $\geq 15$. Participants with Folstein MiniMental State Examination $(\mathrm{MMSE})^{21}$ score of $<24$ or DSM-IV diagnosis of dementia were exclude. In addition, individuals with unstable medical conditions, which may have required treatment with strong anti-inflammatory medications were excluded. Our final sample included 335 individuals who passed clinical and genetic data quality control (see Supplementary Figs. 1-2). The sample included 4,471,676 genotyped (Illumina PsychArray BeadChip) and imputed single-nucleotide polymorphisms (SNPs) at a $5 \%$ minor allele frequency and $99.1 \%$ call rate. All participants provided written, informed consent. The study received ethics approval from institutional review boards at the University of Pittsburgh Medical Center, Washington University School of Medicine, and Centre for Addiction and Mental Health.

\section{Validation cohorts}

To validate any top-associated SNPs, we explored three external cohorts, which included adults treated with 
citalopram from Level 1 of STAR*D ${ }^{22}$, CANBIND- $1^{23}$ and STOP-PD $\mathrm{II}^{24}$. All studies received ethics approval at relevant institutional boards and all participants provided written, informed consent. We applied the same quality control and imputation criteria to all cohorts as described for the IRL-GREY sample.

\section{$S T A R^{*} D$}

We included 821 individuals of European-ancestry from Level 1 of the multi-site, clinical study STAR*D (Sequenced Treatment Alternatives to Relieve Depression; NCT00021528 ${ }^{22}$. Participants were diagnosed with MDD according to DSM-IV criteria and had a baseline Hamilton Depression Rating Scale (HRSD) score of $\geq 14$. Individuals received prospective treatment with citalopram for 8-12 weeks. Genotyping for the sample was conducted by the original authors, with approximately half the samples genotyped on the Affymetrix Human Mapping 500k Array Set and half on the Affymetrix Genome-Wide Human Array 5.0 ${ }^{25}$.

\section{Canadian Biomarker Integration Network for Depression Study (CANBIND-1)}

The CANBIND-1 cohort is a multicentre cohort of individuals diagnosed with MDD receiving treatment for up to 16 weeks ${ }^{23}$. For the first eight weeks, all participants received escitalopram (10-20 mg/day). Individuals who reached remission at eight weeks continued for another eight weeks on escitalopram, which non-remitters received augmentation with aripiprazole $(2-10 \mathrm{mg} /$ day). The full protocol is described in detail in previous publications. Individuals were assessed using the MADRS at nine time-points and were genotyped using the Illumina Omni 2.5 BeadChip $^{26}$. For our investigation on antidepressant response, we used remission status from week eight of treatment before individuals received augmentation with aripiprazole.

\section{Sustaining Remission of Psychotic Depression II (STOP-PD II)}

STOP-PD II was a 36-week randomized clinical trial (RCT) that compared the efficacy and tolerability of sertraline plus olanzapine with sertraline plus placebo in preventing relapse of remitted psychotic depression ${ }^{24}$. Before the RCT, patients aged 18-85 years with unipolar psychotic depression received open-label sertraline (target dose of $150-200 \mathrm{mg} /$ day) and olanzapine (target dose of $15-20 \mathrm{mg} /$ day) for up to 12 weeks of acute treatment. For analysis, remission of depressive symptoms was defined post-hoc as an HRSD total score $\leq 7$, which was the definition used in STAR*D. Individuals were genotyped on the Illumina PsychArray BeadChip at TCAG (Toronto, Canada). We restricted the sample to 114 individuals of European-ancestry who completed the acute phase of the study with non-missing clinical (i.e. age, sex, baseline
HRSD score, final HRSD score) and genotype data (i.e. European principal components and SNP genotypes).

\section{Genome-wide association studies}

Our primary and secondary outcomes of interest were remission status defined as MADRS score $\leq 10$ at the end of treatment (i.e. week 12$)^{27}$ and symptom improvement defined as positive percentage change in MADRS score from baseline to end of treatment, respectively. We chose to prioritize remission as the phenotype of interest despite it being a dichotomized outcome, given that remission is considered a primary outcome of clinical antidepressant treatment ${ }^{28}$. We conducted genome-wide logistic and linear regressions adjusted for sex, recruitment site, age, duration of treatment, duration of the current depressive episode and the first two principal components from standard ancestry analysis (for details, see Supplementary Methods). For remission status, we also included the baseline MADRS score as a covariate.

To validate any associations, we conducted metaanalyses within three validation cohorts (i.e. STOP-PD II, CANBIND-1 and STAR*D). In the case of STAR*D and STOP-PD II, remission was defined as a Hamilton Rating Scale for Depression (HRSD) score $\leq 7^{29}$, whereas, for CANBIND-1, we used similar MADRS score $\leq 10$ criteria as for IRL-GREY (see Supplementary Table 1). Separate associations were first conducted within each cohort, including similar covariates, were available, including sex, age, baseline depressive severity and the first two principal components from ancestry analysis. The meta-analysis was conducted using $M E T A L^{30}$ for variants present in at least one validation cohort. Within METAL, variant effects were combined using an inverse-variance-weighted, average method allowing for random effects, given the heterogeneity across our four cohorts (assessed using the Cochran $\mathrm{I}^{2}$ test for heterogeneity). We chose to use inverse-variance weighting as opposed to weighting by the effective sample size to estimate an averaged effect size (i.e. beta). For the resulting association, we controlled for multiple testing using genomic control (i.e. lambda). Although these cohorts are heterogeneous and include younger adults, validation of our top hits would allow us to understand the generalizability of markers and their associations with venlafaxine response across the adult lifespan.

We further characterized associations using time-toremission (Cox regression using $R$ package survival) ${ }^{31}$ and response trajectories (linear mixed-effects models using $R$ package $l m e 4)^{32}$, including the same covariates (see Supplementary Figs. 3-4). Given that analyses of the smaller African, Asian-Pacific, and admixed samples would have resulted in a loss of power for genome-wide analyses, our primary analyses focused on individuals of confirmed European-ancestry. 


\section{Genome-wide gene analyses}

Genome-wide gene analyses were conducted using $M A G M A^{33}$ as performed using FUMA v1.3.5 $\mathrm{e}^{34}$ with default parameters (SNP-wide mean model). In brief, SNPs were assigned to genes within a $10 \mathrm{~kb}$ window based on a combined reference panel including 1000 Genomes Phase 3 reference panels (2504 individuals, $\sim 84.8$ million SNPs) and a subset of the UK Biobank data $(10,000$ individuals, $\sim 17$ million SNPs) ${ }^{34}$. We further explored whether top-associated genes showed over-representation for (1) tissue-specific, differentially expressed genes (DEGs), (2) known biological pathways, and (3) previous genome-wide significant associations. For gene expression, top-associated genes were compared to existing, precalculated DEGs across 54 GTEx $^{35}$ tissues. Next, we explored geneset over-representation for biological processes, molecular functions, and cellular localization defined by Gene Ontology $(10,192 \text { genesets })^{36}$, as well as canonical pathways from $M s i g D B$, including $K E G G^{37}$, Reactome $^{38}$, BioCarta ${ }^{39}$ and the Pathway Interaction Database $^{40}$. Lastly, top-associated genes were compared to previously associated hits curated by the GWAS cata$\operatorname{logue}^{41}$. To assess over-representation, hypergeometric tests were conducted within FUMA with Bonferronicorrection.

\section{Polygenic risk scores}

Polygenic risk scoring (PRS) was used to evaluate the potentially shared genetic architecture between LLD, neurodegeneration, and cerebrovascular disease. We constructed 11 risk scores for outcomes across six large genome-wide studies, including for depression ${ }^{42-44}$, Alzheimer's disease ${ }^{45,46}$, and various strokes, such as ischemic, cardioembolic, large vessel and small vessel stroke $^{47}$ (see Supplementary Tables). These studies were selected for their large sample sizes (i.e. mega- and metaanalyses) and including publicly available summary statistics from individuals of European-ancestry. For each outcome, scores were constructed using PRSice-2 v.2.2 $2^{48}$ across ten $p$-value thresholds, with lower and upper thresholds of $P_{T}=10^{-4}$ and $P_{T}=1$. The constructed scores were then evaluated for their association with either remission status or percentage symptom improvement using linear and logistic regressions adjusted for ancestry principal components 1 and 2, age, sex, recruitment site, treatment duration, baseline MADRS score and episode duration. To control for multiple testing, we conducted within-score permutation testing across 10,000 resamples and between-score, Bonferroni-correction for 11 scores. To control for Type I error within the process of calculating each PRS, we also conducted permutation testing to obtain an empirical $\mathrm{p}$-value for the best $p$-value threshold $\left(P_{0}\right)$. Ten thousand random phenotype permutations were used to assess the PRS model under the null.

\section{Multiple polygenic risk scores and outcome prediction}

Given the complexity of our traits of interest, we further investigated the joint predictive capacity of combining single polygenic scores, which may capture vulnerabilities across different genetic pathways. The multiple polygenic risk score (mPRS) approach has been shown to capture more genetic variance than single scores potentially ${ }^{49}$. Therefore, we followed a similar approach to building predictive models using elastic net regression. Elastic net regression allows for variable mixing of $\mathrm{L}_{1}$ and $\mathrm{L}_{2}$ shrinkage ${ }^{50}$, thereby potentially allowing for more correlation (e.g. the genetic correlation between polygenic scores) than LASSO regression, but less correlation than ridge regression (i.e. allowing for exclusion of redundant information).

First, PRS scores were calculated at a common $p$-value threshold of 0.05 for all discovery samples to avoid information leakage resulting from fitting the 'best' $p$ value threshold from single polygenic score analysis. After scoring individuals, we constructed the predictive models in R using the caret $^{51}$ and glmnet $^{52}$ packages. The cohort was randomly split into a $70 \%$ training set and a $30 \%$ holdout testing set. Within the training set, three sets of models were constructed for each outcome of interest (i.e. remission and improvement): (1) a null model with a permuted outcome; (2) a clinical model including individual, baseline predictors (i.e. sex, age, baseline MADRS score and MDE duration) and, (3) a full model including the four clinical variables and all polygenic scores. For variable pre-processing, we assessed for excessive correlation $(\rho>0.8)$ and near zero-variance ( $<5 \%$ frequency), as well as included dummy coding for the recruitment site. Note, separate clinical and polygenic models were not constructed to select the best predictors for a final model to avoid selection bias. Furthermore, the elastic net model includes an internal variable selection process, whereby unimportant variables (i.e. penalized coefficients $=0$ ) are excluded from the final model.

Within the training set, alpha and lambda hyperparameters (random search grid) were tuned using 100xrepeated 10-fold cross-validation to optimize the area under the receiver operating curve (AUC) for remission, and root mean square error (RMSE) for percentage symptom improvement. The tuned models (null, clinical, and combined) were then fit on the holdout testing to assess their predictive performance. Models for remission were assessed using AUC, sensitivity, and specificity, while models for percentage improvement were assessed on RMSE, R-squared $\left(R^{2}\right)$, and mean absolute error (MAE). Lastly, to assess the importance of predictors, we retrieved effect sizes (i.e. beta coefficients). The significance of model performance was assessed using accuracy for remission (i.e. one-sided exact proportion test compared 
to null remission prevalence) and two-sided Pearson correlation test for percentage symptom improvement (i.e. predicted symptom improvement compared to observed).

Given that IRL-GREY is a unique cohort of older adults treated with a specific antidepressant, we did not include STOP-PD, CANBIND-1, and STAR"D in the analyses. In particular, previous studies have shown that predictive models may be antidepressant-specific ${ }^{53}$, therefore, we do not expect that these models will generalize to older adults treated with non-SNRIs (e.g. STOP-PD II and STAR $* D$ aged $>60$ ) or younger adults treated with nonSNRIs (e.g. STOP-PD II aged $<60$, STAR*D aged $<60$, CANBIND-1). See Fig. 1 for analysis workflow overview.

\section{Results}

\section{Sample demographics}

Our final sample included 335 individuals who were predominantly of European $(n=307,91.6 \%)$ and African $(n=22,6.6 \%)$ ancestry. While the main results are presented from the European sub-cohort, where appropriate, we also present associations from the African-ancestry sub-cohort and mixed-ancestry, total sample. In the European sub-cohort, individuals were predominantly female $(62.2 \%)$ with a mean age of $68.9(S D=7.0)$ years. In brief, $52.4 \%$ of individuals were classified as remitters at the end of treatment, reaching remission, within 10.3 weeks $(S D=4.7)$. The mean dose of venlafaxine was $241.4 \mathrm{mg} /$ day $(S D=70.8)$ at the end of treatment. For additional details, including summaries for the Africanancestry sub-cohort and total, mixed-ancestry sample, see Supplementary Table 2.

\section{SNP-based GWAS}

In brief, there were no genome-wide significant SNPs in association with remission status or percentage change in the MADRS score by the end of treatment (i.e. symptom improvement). However, there were eight genomic loci at a less conservative, suggestive threshold for significance $\left(p=5 \times 10^{-6}\right)$, which we considered of potential interest for exploration (see Fig. 2). For additional information, see Supplementary Tables 3-12.

For remission status, the top-associated variant was PIEZO1 rs12597726 $(O R=0.33$ [0.21, 0.51], $p=1.42 \times$ $\left.10^{-6}\right)$ which also showed a non-GWAS significance in association with improvement (unstandardized beta $(B)=$ $\left.-14.25[-20.44,-8.06], p=9.33 \times 10^{-6}\right)$. In other words, individuals carrying at least one rs 12597726 effect allele (A) show a $67 \%$ decreased chance of being a remitter or $14.25 \%$ less improvement at the end of treatment. Furthermore, having at least one A-allele was associated with a worse response trajectory $\left(F_{(6,1681)}=4.64, p=1.06 \times\right.$ $10^{-4}$ ) and slower time to remission (Median $=14.3$ weeks) compared to those with a G/G genotype (Median=
11.9 weeks, $95 \%$ C.I. $=[10,13.1])$ after adjusting for covariates $\left(H R=0.58[0.42,0.82], p=1.58 \times 10^{-3}\right.$; see Fig. 3$)$. For model diagnostics, see Supplementary Figs. 3-4.

Although the African-ancestry sub-cohort had a markedly low minor allele frequency for rs 12597726 (3\%) compared to the European-Ancestry group, in the total, mixed-ancestry sample, rs12597726 showed a similar effect (see Supplementary Table 3). Furthermore, we observed a similar directionality of association in the STOP-PD II and CANBIND-1 cohorts, which included the variant, under the random-effects models weighted both by the standard error $\left(O R=0.51, p=1.48 \times 10^{-4}\right)$ and sample size $\left(N_{\text {eff }}=474.16, Z=-3.96, p=7.46 \times\right.$ $10^{-5}$ ) after genomic control. Of note, the rs 12597726 is an annotated regulatory feature with a predicted functionality CADD score of 12.4 , suggesting that rs 12597726 is among at least the $10 \%$ most deleterious substitutions in the human genome. While rs12597726 does not appear to affect PEIZO1 expression in brain tissue, rs12597726 shows the strongest effect on expression within the muscularis mucosae of the esophagus where the A-allele is associated with higher PIEZO1 expression (GTEx expression, normalized effect size $=0.15, p=$ $9.4 \times 10^{-5}$; see Supplementary Figs. 5-6).

For improvement, the top-associated variant was rs6916777 $\left(B=14.03\right.$ [8.47, 19.59], $\left.p=1.25 \times 10^{-6}\right)$, an intronic variant in the non-coding RNA, RP11-510H23.1. Of note, rs6916777 was also one of three variants passing the suggestive threshold for remission status $(O R=2.58$ $\left.[1.73,3.85], p=3.53 \times 10^{-6}\right)$. Having at least one A-allele was associated with a better response trajectory $\left(F_{(6,1676)}=\right.$ 4.36, $p=1.79 \times 10^{-3}$ ) and faster time to remission (Median $=13.0)$ compared to those with a $\mathrm{C} / \mathrm{C}$ genotype (Median $=16.0$ weeks) after adjusting for covariates $(H R=$ 1.61 [1.10, 2.36], $p=0.01$ ). Specifically, those with the A/A genotype reach remission at a median time of 10.4 weeks $(95 \%$ C.I. $=[10.4,7.86])$.

Although it is predicted that rs6916777 is unlikely to be functional (CADD PHRED $=0.54$ ), carriers of the A-allele in IRL-GREY were $158 \%$ more likely to be remitters and had $14.03 \%$ greater reduction in depressive severity compared to $\mathrm{C} / \mathrm{C}$ genotypes. However, we observed a significant, opposite effect of rs6916777 in the Africanancestry cohort $(B=-26.03[-44.75,-7.31], p=0.016)$ despite a similar minor allele frequency. Furthermore, no effect of rs6916777 was found in the meta-analysis of the four cohorts under either weighting scheme (by the standard error, $B=2.22, p=0.13$; by sample size, $N_{\text {eff }}=$ 1325.65, $Z=1.09, p=0.27$ ).

\section{Gene-based GWAS}

Subsequently, we conducted genome-wide gene-based associations with remission status and percentage symptom improvement using MAGMA. The input SNPs 


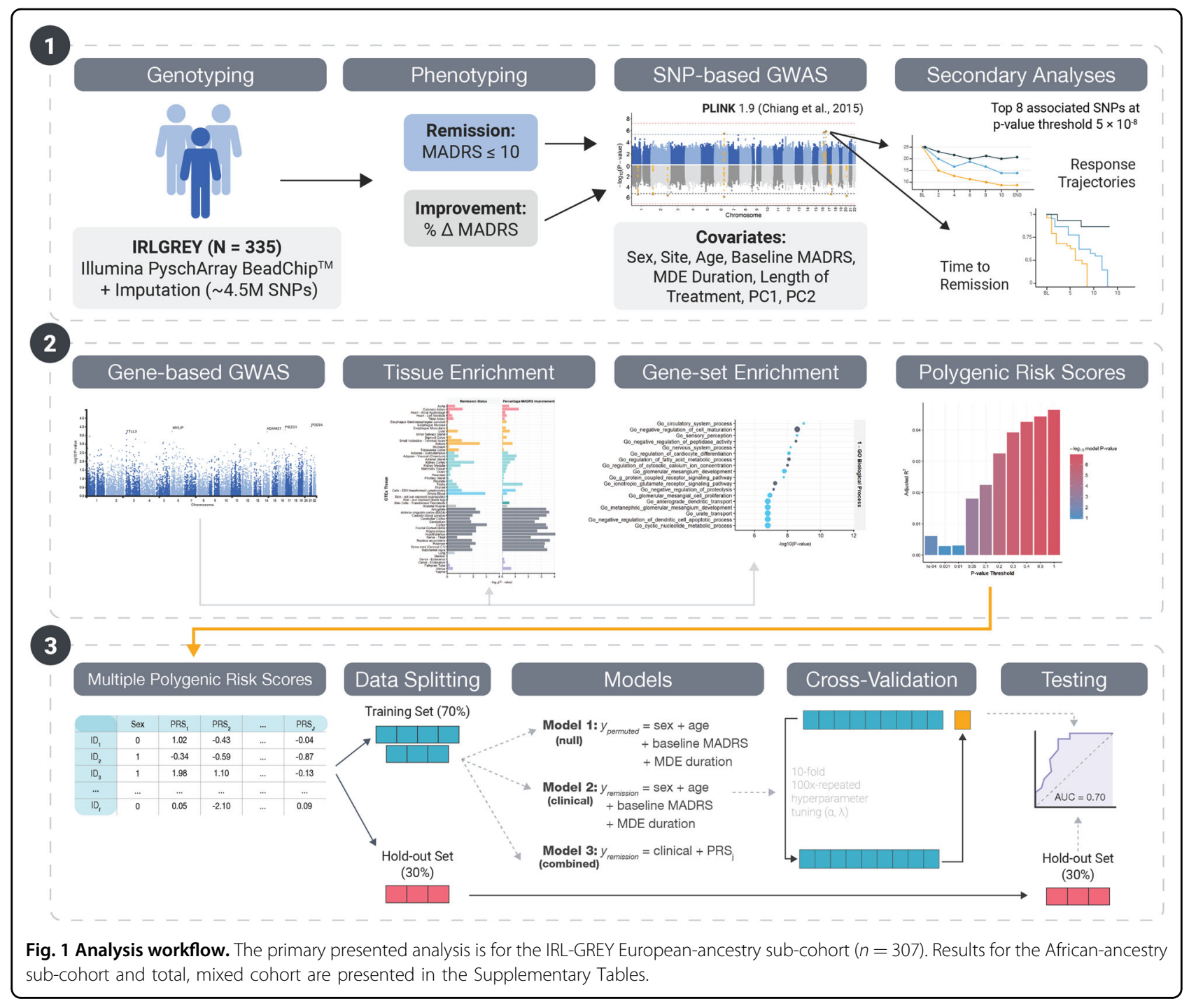

were mapped to 17,748 protein-coding genes. No genes reached genome-wide significance after Bonferronicorrection (i.e. $\alpha=0.05 / 17,748$ genes $=2.82 \times 10^{-6}$ ) for remission status or percentage symptom improvement (see Supplementary Table 13). PDE9A (Z-score $=3.71$, $\left.p=1.05 \times 10^{-4}\right)$ and PIEZO1 $\left(Z=3.59, p=1.60 \times 10^{-4}\right)$ were the top two genes associated with remission status, while FPR3 $\left(Z=3.73, p=9.55 \times 10^{-5}\right)$ and GRIK4 $(Z=$ $3.55, \quad p=1.90 \times 10^{-4}$ ) were top associations with improvement. Of note, PIEZO1 also showed a top association with improvement $(Z=3.48, p=2.51 \times$ $10^{-4}$ ). We saw added support for the SNP-based association for rs6916777 with RNF217, with RNF217 being among the top ten genes associated with improvement $\left(Z=3.37, p=3.77 \times 10^{-4}\right)$. In addition, we extracted 51 genes from the literature that have been previously associated with antidepressant response in MDD and LLD (see Supplementary Table 14). Across the two outcomes of interest, we observed associations of GRIK4 as a top-hit and SLC6A2 $\left(Z\right.$-score $_{\text {Remission }}=2.47, p=$ $6.69 \times 10^{-3}, Z$-score Improvement $\left.=3.55, p=0.023\right)$.

\section{Geneset and tissue enrichment}

We did not observe any evidence of specific tissue enrichment for remission status or symptom improvement (see Fig. 4 and Supplementary Table 15). However, we observed a significant association of two highlyoverlapping pathways for peptidase regulator activity with symptom improvement $(n=25$ of 190 genes, $p=$ $8.03 \times 10^{-6}$, Benjamini-Hochberg FDR-corrected $p=$ $0.01)$. For remission status, the top-associated geneset prior to FDR correction was the GO pathway for circulatory system processes $(n=43$ of 479 genes, $p=1.26 \times$ $10^{-4}$, FDR-corrected $p=0.51$; see Supplementary Tables 16-17). In addition, the top-associated GWAS Catalog genesets were for cardiac structure and function $(n=4$ of 


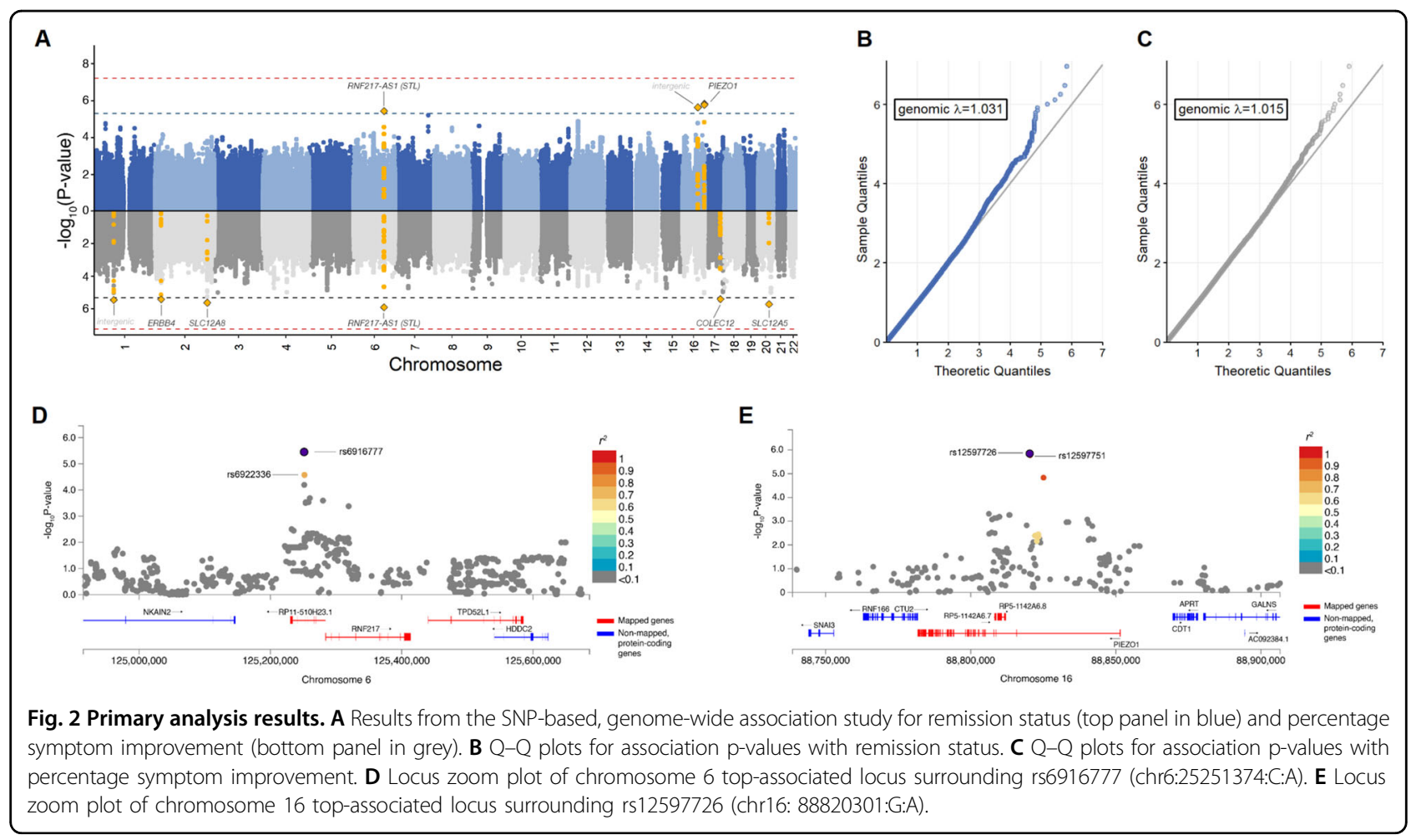

6 genes, $p=8.30 \times 10^{-5}$, FDR-corrected $p=0.15$; see Supplementary Table 18).

\section{Polygenic risk scores}

The PRS for cardioembolic stroke was significantly associated with remission status and symptom improvement, which remained significant after permutation testing and Bonferroni-correction $(\alpha=0.0045)$ for the 11 PRS scores that were built (see Fig. 5 and Supplementary Tables 19-20). The cardioembolic stroke PRS reached significance at a $p$-value threshold of 0.05 and achieved the highest explained variance (adjusted $R^{2}=0.046$ ) at the most lenient $p$-value threshold, including 75,508 SNPs. In a full model, a $1 \mathrm{SD}$ increase in polygenic risk for cardioembolic stroke, was associated with decreased probability of remission $(O R=0.63[0.48,0.83], p=0.001$, permutation $p=0.006)$ or $5.51 \%$ less improvement (MADRS, beta $=-5.51[-9.45$, -1.57], $p=0.01$, permutation $p=0.033)$. Overall, the addition of polygenic risk for cardioembolic stroke improved the model fit for remission as compared to a model with only clinical variables (likelihood ratio test, $\left.\chi_{(1)}^{2}=1.21, p=4.78 \times 10^{-4}\right)$, as well as marginally for improvement $\left(\chi_{(1)}^{2}=3.78, p=0.052\right)$.

\section{Predicting treatment outcomes using mPRS}

Given the association of the polygenic risk for cardioembolic stroke with treatment non-remission and less symptom improvement, we evaluated the predictive capacity of the 11 risk scores when added to a clinical model.
For remission status, both models showed significant predictive performance with the mPRS model achieving marginally better performance $(A U C=0.70$, Sensitivity $=$ 0.72 , Specificity $=0.67 ;$ Accuracy $=0.70[0.59,0.79], p=$ $\left.2.45 \times 10^{-4}\right)$ compared to the clinical model $(A U C=0.70$, Sensitivity $=0.64$, Specificity $=0.71 ;$ Accuracy $=0.67[0.57$, 0.77], $p=1.13 \times 10^{-3}$; see Supplementary Tables 21-22). In the full model, the top five predictors included sex (100\% importance), risk for ischemic stroke $(60.9 \%$ importance compared to sex), risk for cardioembolic stroke (48.9\%), MDE duration (38\%) and risk for large vessel stroke (30.8\%; see Fig. 5). However, for percentage improvement, neither the mPRS model $\left(R M S E=36.93, R^{2}\right.$ $=0.04, M A E=31.20$; Pearson's $\rho=0.19$ [-0.02, 0.36], $p=$ $0.07)$ nor the clinical model ( $R M S E=37.43, R^{2}=0.03$, $M A E=31.73$; Pearson's $\rho=0.16[-0.04,0.36], p=0.12$ ) was significant.

\section{Discussion}

This is the first genome-wide study of venlafaxine response in older adults, with our sample being the largest cohort of LLD patients with genome-wide SNP and clinical data. Although we did not observe any genomewide significant association with remission status or percentage symptom improvement, it remains important to evaluate suggestive findings in the context of previous studies to begin generating further hypotheses for investigation and to explore if already-known processes are involved in response. 
A

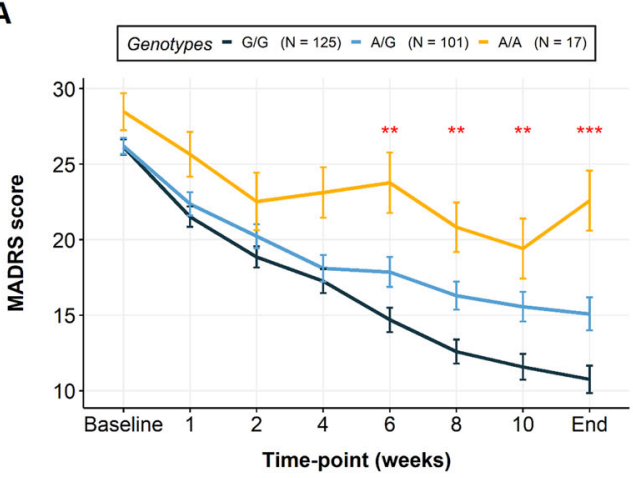

C
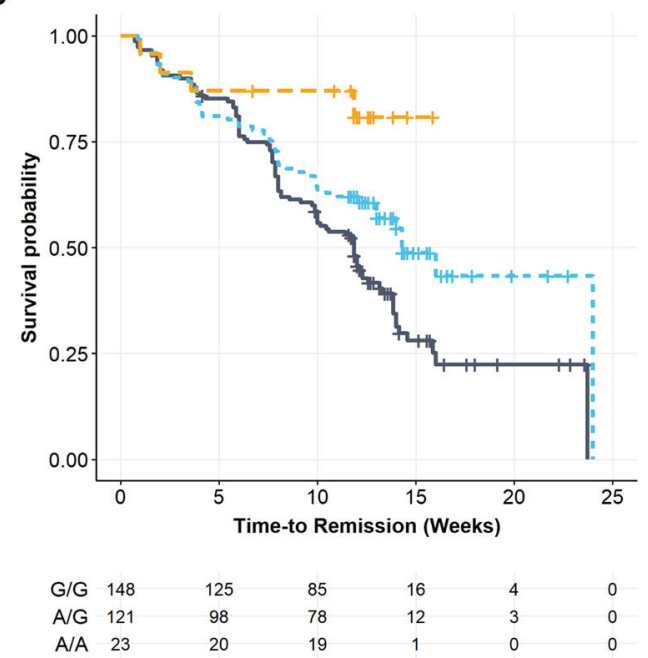

B

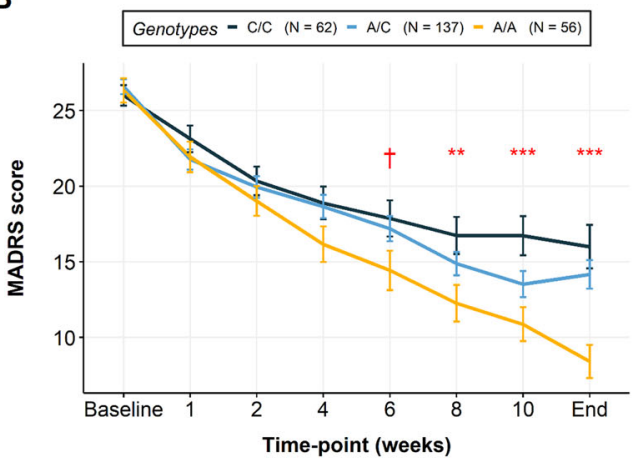

D

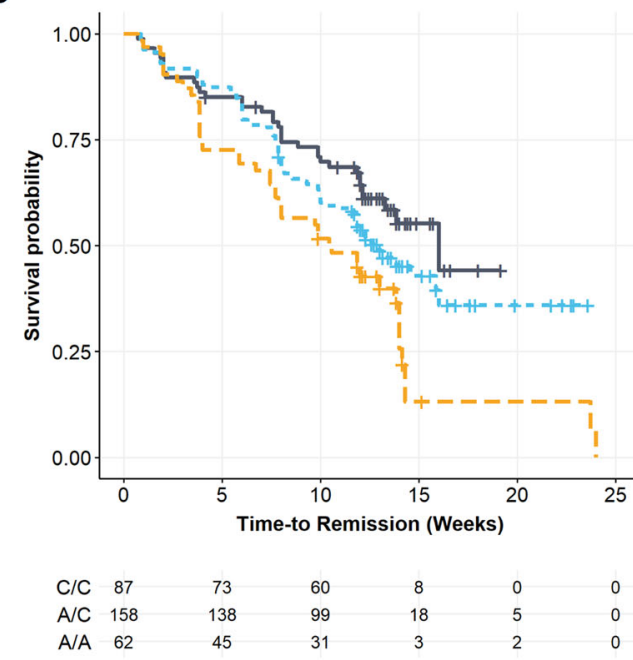

Fig. 3 Secondary analysis results for top-associated SNPs, rs6916777 (chr6:25251374:C:A) and rs12597726 (chr16: 88820301:G:A).

A, B Mixed-effects analyses for rs6916777 and rs12597726, respectively. Values at each time-point denote mean per genotype, and error bars denote standard error of the mean. C, D Kaplan-Meier survival plots for rs6916777 and rs12597726, respectively. Risk tables denote the number of nonremitted or censored individuals at each time-point. Significance levels. ${ }^{* *} p<0.001,{ }^{* *} p<0.01,{ }^{*} p<0.05,{ }^{\dagger} p<0.1$.

Our top association with remission status was rs12597726 in the PIEZO1 gene, which encodes for Piezo Type Mechanosensitive Ion Channel Component 1. PIEZO1 channels are critical for vascular remodelling, including angiogenesis, and have implications for hypertension, aneurysms and stroke $\mathrm{e}^{54-57}$. These channels prevent microglial activation by pro-inflammatory cytokines and chemokines, including IL- $1 \beta$ and TNF- $\alpha$, in response to neurodegenerative amyloid-beta plaques and ischemic events $^{58-61}$. Despite generally low PIEZO1 expression levels in the brain, the rs12597726 A-allele is associated with lower PIEZO1 expression, which may ultimately contribute to higher inflammation and non-response (see Supplementary Figs. 5-6). Overall, our finding that increased vascular risk is associated with worse response to venlafaxine in our sample support implicated mechanisms and the vascular depression hypothesis ${ }^{11}$.

Our findings also implicate the ubiquitin-proteasome system, which is involved in intracellular protein degradation. Specifically, we observed that the intergenic variant rs6916777 showed the strongest association with symptom improvement. Although intergenic, rs6916777 is a cis-eQTL for the downstream gene RNF217 (Ring Finger Protein 217) across multiple tissues (see Supplementary Figs. 7-8). Although the function of RNF217 remains unclear, RNF217 facilitates B-cell maintenance processes, primarily apoptosis through the ubiquitinproteasome system ${ }^{62}$. In the IRL-GREY cohort, the rs6916777 A-allele was associated with better venlafaxine response, which may be due to lower expression of RNF217. This finding supports previous evidence of an association between the ubiquitin-proteasome system and antidepressant response ${ }^{63}$.

For the gene-based association study, there were no genome-wide significant genes; however, we observed whole-gene associations which echoed single-SNP results. PIEZO1 showed a nominal association, as well as RNF217. Other top gene associations included PDE9A for 


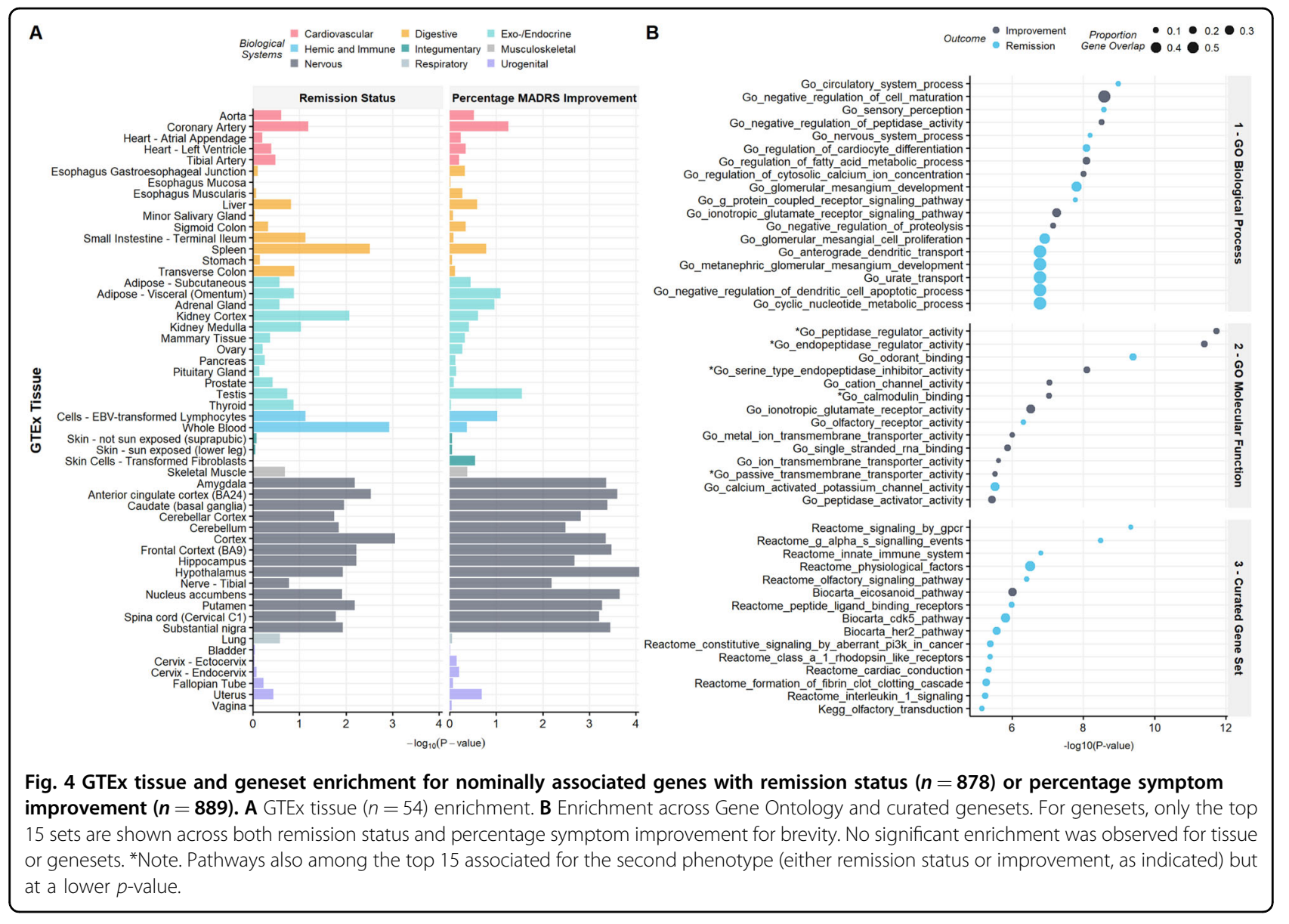

remission, as well as FPR3 and GRIK4 for symptom improvement. GRIK4 encodes for the Kainate-type Ionotropic Glutamatergic Receptor Subunit 4, which has been associated with citalopram response in the STAR*D cohort $^{64}$. Similarly, variants in the gene encoding for the Phosphodiesterase $9 \mathrm{~A}$ enzyme (PDE9A) have been associated with MDD risk but not response across various antidepressants, including fluoxetine, desipramine, and citalopram $^{65,66}$. GRIK4 and PDE9A contribute to critical pathways involved in depression and antidepressant response, including glutamatergic signalling, neuroplasticity and neurogenesis ${ }^{67}$. Unlike GRIK4 and PDE9A, the function of FPR3 remains unclear but shares $83 \%$ of its sequence with FPR2, which has been implicated in neuroinflammation via microglial activation ${ }^{68,69}$. Nonetheless, among these proteins, PDE9A is a druggable-target, which is currently in clinical trials for Alzheimer's disease, while FPRs have been postulated as possible targets for mitigating ischemia-induced inflammation ${ }^{70,71}$.

In addition, we explored the polygenic overlaps between venlafaxine response in late-life, all-age depression, Alzheimer's disease and stroke. While we observed several nominal associations, only the polygenic overlap between venlafaxine non-response and increased risk for cardioembolic and large vessel stroke remained significant. Although it is difficult to disentangle the genetic effects critical to confluent pathways involved in depression, neurodegeneration and cerebrovascular disease, further investigation is required ${ }^{3}$.

The main limitation of this investigation is the relatively small sample size. Given that we selected the dichotomized variable- remission as our outcome, the analysis suffered a loss of power, which may have led to a lack of significantly associated variants. Therefore, we attempted to validate any putative associations in three external cohorts of adults treated with antidepressants. However, these cohorts were heterogeneous in age and venlafaxine treatment, which did not allow for replication. Notably, the validation cohorts included treatment with SSRIs that may have different pharmacogenetic contributions than venlafaxine, a dual serotonin-norepinephrine reuptake inhibitor. While we also observed a significant predictive effect of polygenic risk scores for cardioembolic and large vessel stroke, overall, the improvement in performance compared to a base clinical model was minimal. However, due to the polygenicity of a complex outcome such as antidepressant response, variants likely contribute small effects and the addition of other risk scores, for example, 

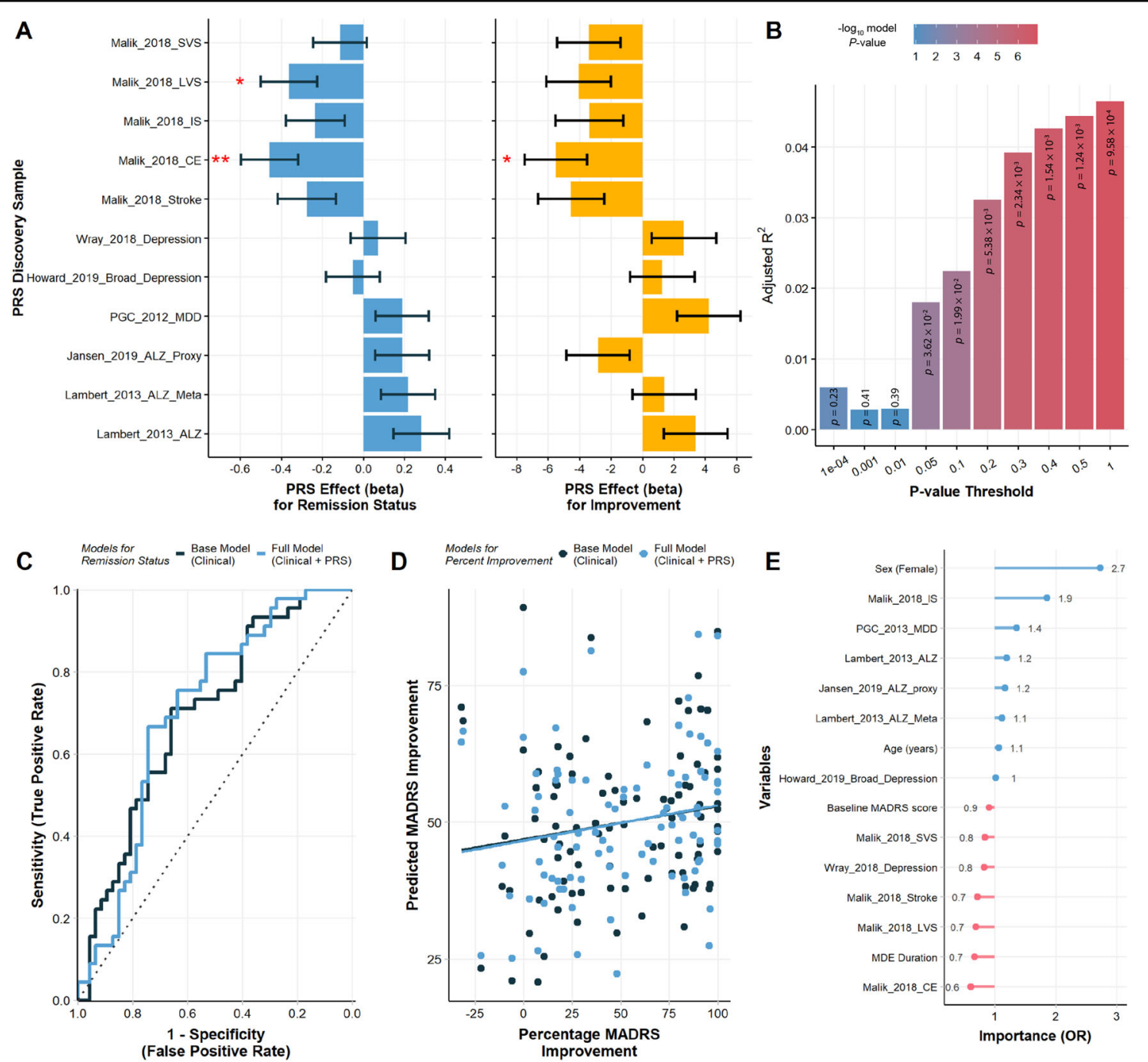

Fig. 5 Polygenic risk scores and prediction. A Polygenic risk score effect sizes for remission status (blue, odds ratios) and percentage symptom improvement (yellow, unstandardized betas). Error bars denote standard error of the mean. B Variance in remission status explained by the polygenic risk score regression results for cardioembolic risk (Malik et al., 2018). C, D Receiver operating curves for prediction of $\mathbf{C}$ remission status and D symptom improvement comparing a base clinical model to a model including the 11 polygenic risk scores. E Variable importance from the best performing model for remission status.

for other psychiatric, neurodegenerative and cardiovascular outcomes, may improve performance.

In the context of existing literature, our results underline the importance of neuroinflammation, as well as vascular health and its consideration in venlafaxine treatment in older adults. Among the genes identified, such as PDE9A, there is a potential to inform druggable targets and further exploration of drug repurposing and treatment development to address neuroinflammatory and vascular pathways. However, further investigations will require more extensive and pooled samples to increase the power to detect non-spurious associations with small effects. Overall, these findings elucidate contributions to venlafaxine treatment response, particularly in older adults who may have unique pharmacokinetic and pharmacodynamic characteristics.

\section{Acknowledgements}

This work was supported by Canadian Institutes for Health Research.

\section{Author details}

${ }^{1}$ Institute of Medical Science, University of Toronto, Toronto, ON, Canada. ${ }^{2}$ Campbell Family Mental Health Research Institute, Centre for Addiction and Mental Health, Toronto, ON, Canada. ${ }^{3}$ Center of Experimental Rheumatology, Department of Rheumatology, University Hospital Zurich, University of Zurich, Zurich, Switzerland. ${ }^{4}$ Medical Bioinformatics, University of Marburg, Marburg, Germany. ${ }^{5}$ Department of Pharmacology \& Toxicology, University of Toronto, Toronto, ON, Canada. ${ }^{6}$ Department of Psychiatry, University of Toronto, Toronto, ON, Canada. ${ }^{7}$ Department of Psychiatry, University of Pittsburgh, Pittsburgh, PA, USA. ${ }^{8}$ Centre for Mental Health, University Health Network, Toronto, ON, Canada. ${ }^{9}$ McGill Group for Suicide Studies, Douglas Mental Health 
University Institute, McGill University, Verdun, QC, Canada. ${ }^{10}$ University of British Columbia and Vancouver Coastal Health Authority, Vancouver, BC, Canada. "'Department of Psychiatry, Queen's University, Kingston, ON, Canada. ${ }^{12}$ Department of Psychiatry and Behavioural Neurosciences, McMaster University, Hamilton, ON, Canada. ${ }^{13}$ St. Joseph's Healthcare Hamilton, Hamilton, ON, Canada. ${ }^{14}$ Keenan Research Centre for Biomedical Science, Li Ka Shing Knowledge Institute, St Michael's Hospital, Toronto, ON, Canada. ${ }^{15}$ Department of Psychiatry, St Michael's Hospital, University of Toronto, Toronto, ON, Canada. ${ }^{16}$ Healthy Mind Lab, Department of Psychiatry, Washington University, St. Louis, MO, USA

\section{Conflict of interest}

E.J.L. has received funding from Takeda, Lundbeck, Janssen, Alkermes, Aptynx, and PCORI, and is a consultant for Janssen and Jazz Pharmaceuticals. B.H.M. currently receives research support from Brain Canada, the Canadian Institutes of Health Research, the CAMH Foundation, the Patient-Centered Outcomes Research Institute (PCORI), the US National Institute of Health (NIH), Capital Solution Design LLC (software used in a study founded by CAMH Foundation), and HAPPYneuron (software used in a study founded by Brain Canada). He directly owns stocks of General Electric (less than $\$ 5,000$ ). Within the past 3 years, he has also received research support from Eli Lilly (medications for an $\mathrm{NIH}$-funded clinical trial) and Pfizer (medications for an NIH-funded clinical trial).

\section{Publisher's note}

Springer Nature remains neutral with regard to jurisdictional claims in published maps and institutional affiliations.

Supplementary information The online version contains supplementary material available at https://doi.org/10.1038/s41398-021-01248-3.

Received: 18 November 2020 Revised: 15 December 2020 Accepted: 7 January 2021

Published online: 15 February 2021

\section{References}

1. Byers, A. L., Yaffe, K., Covinsky, K. E., Friedman, M. B. \& Bruce, M. L. High occurrence of mood and anxiety disorders among older adults: The National Comorbidity Survey Replication. Arch. Gen. Psychiatry 67, 489-496 (2010).

2. Whyte, E. M. et al. Geriatric depression treatment in nonresponders to selective serotonin reuptake inhibitors. J. Clin. Psychiatry 65, 1634-1641 (2004).

3. Naismith, S. L., Norrie, L. M., Mowszowski, L. \& Hickie, I. B. The neurobiology of depression in later-life: clinical, neuropsychological, neuroimaging and pathophysiological features. Prog. Neurobiol. 98, 99-143 (2012).

4. Forester, B. P. I. et al. Combinatorial pharmacogenomic testing improves outcomes for older adults With depression. Am. J. Geriatr. Psychiatry https://doi. org/10.1016/j.jagp.2020.05.005 (2020).

5. Mangoni, A. A. \& Jackson, S. H. D. Age-related changes in pharmacokinetics and pharmacodynamics: basic principles and practical applications. Br. J. Clin. Pharmacol. 57, 6-14 (2004).

6. Marshe, V. S. et al. Pharmacogenetic implications for antidepressant pharmacotherapy in late-life depression: a systematic review of the literature for response, pharmacokinetics and adverse drug reactions. Am. J. Geriatr. Psychiatry 28, 609-629 (2020).

7. Karrer, T. M., McLaughlin, C. L., Guaglianone, C. P. \& Samanez-Larkin, G. R. Reduced serotonin receptors and transporters in normal aging adults: a metaanalysis of PET and SPECT imaging studies. Neurobiol. Aging 80, 1-10 (2019).

8. Ren, F. et al. Pharmacogenetic association of bi- and triallelic polymorphisms of SLC6A4 with antidepressant response in major depressive disorder. J. Affect. Disord. 273, 254-264 (2020).

9. Charlson, M. \& Peterson, J. C. Medical comorbidity and late life depression: what is known and what are the unmet needs? Biol. Psychiatry 52, 226-235 (2002).

10. Oslin, D. W. et al. Association between medical comorbidity and treatment outcomes in late-life depression. J. Am. Geriatr. Soc. 50, 823-828 (2002).

11. Butters, M. A. et al. Pathways linking late-life depression to persistent cognitive impairment and dementia. Dialogues Clin. Neurosci. 10, 345-357 (2008).
12. Baune, B. T. In Inflammation and Immunity in Depression (ed. Baune BT) Chapter 31, pp 539-548. (Academic Press, 2018).

13. Köhler-Forsberg, O. \& Benros, M. E. in Inflammation and Immunity in Depression (ed. Baune B. T.) Chapter 30, pp 525-538. (Academic Press, 2018).

14. Paradise, M. B., Naismith, S. L., Norrie, L. M., Graeber, M. B. \& Hickie, I. B. The role of glia in late-life depression. Int. Psychogeriatr. 24, 1878-1890 (2012).

15. Navarro, V. et al. Microglia in Alzheimer's disease: activated, dysfunctional or degenerative. Front. Aging Neurosci. https://doi.org/10.3389/fnagi.2018.00140 (2018).

16. Taylor, W., Aizenstein, H. J. \& Alexopoulos, G. S. The vascular depression hypothesis: mechanisms linking vascular disease with depression. Mol. Psychiatry https://doi.org/10.1038/mp.2013.20 (2013).

17. Salo, K. I., Scharfen, J., Wilden, I. D., Schubotz, R. I. \& Holling, H. Confining the concept of vascular depression to late-onset depression: a meta-analysis of MRI-defined hyperintensity burden in major depressive disorder and bipolar disorder. Front. Psychol. 10, 1241 (2019).

18. Szałach, Ł. P., Lisowska, K. A. \& Cubała, W. J. The influence of antidepressants on the immune system. Arch. Immunol. Ther. Exp. 67, 143-151 (2019).

19. Lenze, E. J. et al. Efficacy, safety, and tolerability of augmentation pharmacotherapy with aripiprazole for treatment-resistant depression in late life: a randomised, double-blind, placebo-controlled trial. Lancet 386, 2404-2412 (2015).

20. Montgomery, S. A. \& Asberg, M. A new depression scale designed to be sensitive to change. Br. J. Psychiatry 134, 382-389 (1979).

21. Folstein, M. F., Folstein, S. E. \& McHugh, P. R. "Mini-mental state": a practical method for grading the cognitive state of patients for the clinician. J. Psychiatr. Res. 12, 189-198 (1975).

22. Trivedi, M. H. et al. Evaluation of outcomes with citalopram for depression using measurement-based care in $\operatorname{STAR}^{*} \mathrm{D}$ : implications for clinical practice. Am. J. Psychiatry 163, 28-40 (2006).

23. Kennedy, S. H. et al. Symptomatic and functional outcomes and early prediction of response to escitalopram monotherapy and sequential adjunctive aripiprazole therapy in patients with major depressive disorder: a CAN-BIND-1 report. J. Clin. Psychiatry https://doi.org/10.4088/JCP.18m12202 (2019).

24. Flint, A. J. et al. Effect of continuing olanzapine vs placebo on relapse among patients with psychotic depression in remission: the STOP-PD || randomized clinical trial. JAMA 322, 622-631 (2019).

25. Adkins, D. et al. Genome-wide pharmacogenomic study of citalopraminduced side effects in STAR*D. Transl. Psychiatry https://doi.org/10.1038/ tp.2012.57 (2012)

26. Lam, R. W. et al. Discovering biomarkers for antidepressant response: protocol from the Canadian biomarker integration network in depression (CAN-BIND) and clinical characteristics of the first patient cohort. BMC Psychiatry 16, 105 (2016).

27. Zimmerman, M., Posternak, M. A. \& Chelminski, I. Derivation of a definition of remission on the Montgomery-Asberg depression rating scale corresponding to the definition of remission on the Hamilton rating scale for depression. $J$. Psychiatry Res. 38, 577-582 (2004).

28. Keller, M. B. Remission versus response: the new gold standard of antidepressant care. J. Clin. Psychiatry 65(Suppl 4), 53-59 (2004).

29. Hamilton, M. in Assessment of Depression (eds. Sartorius N., Ban T. A) pp 143-152. (Springer Berlin Heidelberg, 1986).

30. Willer, C. J., Li, Y. \& Abecasis, G. R. METAL: fast and efficient meta-analysis of genomewide association scans. Bioinformatics 26, 2190-2191 (2010).

31. Therneau, T. M. A Package for Survival Analysis in R. https://CRAN.R-project.org/ package $=$ survival (2020).

32. Bates, D., Mächler, M., Bolker, B. \& Walker, S. Fitting linear mixed-effects models using Ime4. J. Stat. Softw. 67, 1-48 (2015).

33. de Leeuw, C. A., Mooij, J. M., Heskes, T. \& Posthuma, D. MAGMA: generalized gene-set analysis of GWAS data.PLoS Comput. Biol. 11, e1004219 (2015).

34. Watanabe, K., Taskesen, E., van Bochoven, A. \& Posthuma, D. Functional mapping and annotation of genetic associations with FUMA. Nat. Commun. $\mathbf{8}$, 1826 (2017).

35. Lonsdale, J. et al. The Genotype-Tissue Expression (GTEx) project. Nat. Genet. 45, 580-585 (2013).

36. Ashburner, M. et al. Gene Ontology: tool for the unification of biology. Nat. Genet. 25, 25 (2000).

37. Kanehisa, M., Sato, Y., Kawashima, M., Furumichi, M. \& Tanabe, M. KEGG as a reference resource for gene and protein annotation. Nucleic Acids Res. 44, D457-D462 (2016). 
38. Jassal, B. et al. The reactome pathway knowledgebase. Nucleic Acids Res. 48, D498-D503 (2020).

39. Nishimura, D. BioCarta. Biotech. Softw. Internet Rep. 2, 117-120 (2001).

40. Schaefer, C. F. et al. PID: the Pathway Interaction Database. Nucleic Acids Res. 37, D674-D679 (2009).

41. Buniello, A. et al. The NHGRI-EBI GWAS Catalog of published genome-wide association studies, targeted arrays and summary statistics 2019. Nucleic Acids Res. 47, D1005-D1012 (2019).

42. Major Depressive Disorder Working Group of the Psychiatric GWAS Consortium. et al. A mega-analysis of genome-wide association studies for major depressive disorder. Mol. Psychiatry 18, 497-511 (2013).

43. Wray, N. R. et al. Genome-wide association analyses identify 44 risk variants and refine the genetic architecture of major depression. Nat. Genet. 50, 668-681 (2018).

44. Howard, D. M. et al. Genome-wide association study of depression phenotypes in UK Biobank identifies variants in excitatory synaptic pathways. Nat. Commun. 9, 1470 (2018)

45. Lambert, J. C. et al. Meta-analysis of 74,046 individuals identifies 11 new susceptibility loci for Alzheimer's disease. Nat. Genet. 45, 1452-1458 (2013).

46. Jansen, I. E. et al. Genome-wide meta-analysis identifies new loci and functional pathways influencing Alzheimer's disease risk. Nat. Genet. 51, 404-413 (2019).

47. Malik, R. et al. Multiancestry genome-wide association study of 520,000 subjects identifies 32 loci associated with stroke and stroke subtypes. Nat. Genet. 50, 524-537 (2018).

48. Choi, S. W. \& O'Reilly, P. F. PRSice-2: polygenic risk score software for biobankscale data. Gigascience 8, giz082 (2019).

49. Krapohl, E. et al. Multi-polygenic score approach to trait prediction. Mol. Psychiatry 23, 1368-1374 (2018).

50. Zou, H. \& Hastie, T. Regularization and variable selection via the elastic net. J. R. Stat. Soc. 67, 301-320 (2005).

51. Kuhn, M. Caret package. J. Stat. Softw. 28, 1-26 (2008).

52. Friedman, J., Hastie, T. \& Tibshirani, R. Regularization paths for generalized linear models via coordinate descent. J. Stat. Softw. 33, 1-22 (2010).

53. Iniesta, R. et al. Antidepressant drug-specific prediction of depression treatment outcomes from genetic and clinical variables. Sci. Rep. 8, 5530 (2018).

54. Li, J. et al. Piezo1 integration of vascular architecture with physiological force. Nature 515, 279-282 (2014).

55. Kang, $\mathrm{H}$. et al. Piezo1 mediates angiogenesis through activation of MT1-MMP signaling. Am. J. Physiol. Cell Physiol. 316, C92-C103 (2019).

56. Beech, D. J. \& Kalli, A. C. Force sensing by Piezo channels in cardiovascular health and disease. Arterioscler. Thromb. Vasc. Biol. 39, 2228-2239 (2019).
57. Hyman, A. J., Tumova, S. \& Beech, D. J. Piezol channels in vascular development and the sensing of shear stress. Curr. Top. Membr. 79, 37-57 (2017).

58. Velasco-Estevez, M. et al. Infection augments expression of mechanosensing Piezo1 channels in amyloid plaque-reactive astrocytes. Front. Aging Neurosci. 10, 332 (2018).

59. Velasco-Estevez, M., Rolle, S. O., Mampay, M., Dev, K. K. \& Sheridan, G. K. Piezo1 regulates calcium oscillations and cytokine release from astrocytes. Glia $\mathbf{6 8}$, 145-160 (2020).

60. Satoh, $K$. et al. A novel membrane protein, encoded by the gene covering KIAA0233, is transcriptionally induced in senile plaque-associated astrocytes. Brain Res. 1108, 19-27 (2006).

61. Sochocka, M., Zwolińska, K. \& Leszek, J. The infectious etiology of Alzheimer's disease. Curr. Neuropharmacol. 15, 996-1009 (2017).

62. Fontanari Krause, L. M. et al. Identification and characterization of OSTL (RNF217) encoding a RING-IBR-RING protein adjacent to a translocation breakpoint involving ETV6 in childhood ALL. Sci. Rep. 4, 6565 (2014).

63. Minelli, A. et al. Proteasome system dysregulation and treatment resistance mechanisms in major depressive disorder. Transl. Psychiatry 5, e687 (2015).

64. Paddock, S. et al. Association of GRIK4 with outcome of antidepressant treatment in the STAR*D cohort. Am. J. Psychiatry 164, 1181-1188 (2007).

65. Wong, M. L. et al. Phosphodiesterase genes are associated with susceptibility to major depression and antidepressant treatment response. Proc. Natl Acad. Sci. USA 103, 15124-15129 (2006).

66. Cabanero, M., Laje, G., Detera-Wadleigh, S. \& McMahon, F. J. Association study of phosphodiesterase genes in the Sequenced Treatment Alternatives to Relieve Depression sample. Pharmacogenet. Genomics 19, 235-238 (2009).

67. Reierson, G. W., Guo, S., Mastronardi, C., Licinio, J. \& Wong, M. L. cGMP sig naling, phosphodiesterases and major depressive disorder. Curr. Neuropharmacol. 9, 715-727 (2011).

68. Cussell, P. J. G., Gomez Escalada, M., Milton, N. G. N. \& Paterson, A. W. J. The Nformyl peptide receptors: contemporary roles in neuronal function and dysfunction. Neural Regen. Res. 15, 1191-1198 (2020).

69. Rogers, J., Mastroeni, D., Leonard, B., Joyce, J. \& Grover, A. In International Review of Neurobiology pp 235-246 (Academic Press, 2007).

70. Kroker, K. S. et al. Inhibition of acetylcholinesterase and phosphodiesterase-9A has differential effects on hippocampal early and late LTP. Neuropharmacology 62, 1964-1974 (2012)

71. Gavins, F. N. E. Are formyl peptide receptors novel targets for therapeutic intervention in ischaemia-reperfusion injury? Trends Pharmacol. Sci. 31, 266-276 (2010). 